\title{
Towards a high efficiency amplifier based on Raman amplification
} \\ G Vieux ${ }^{1}$, E Brunetti ${ }^{1}$, S Cipiccia ${ }^{2}$, B Eliasson ${ }^{1}$, B Ersfeld ${ }^{1}$, \\ J P Farmer ${ }^{3}$, M S Hur ${ }^{4}$, N Lemos ${ }^{5}$, G H Welsh ${ }^{1}$, \\ S M Wiggins ${ }^{1}$, X Yang ${ }^{6}$, S R Yoffe ${ }^{1}$, J M Dias ${ }^{7}$, \\ D A Jaroszynski ${ }^{1}$ \\ ${ }^{1}$ Department of Physics, SUPA and University of Strathclyde, Glasgow, G4 0NG, \\ UK \\ ${ }^{2}$ Diamond Light Source, Harwell Science and Innovation Campus, Didcot, OX11 \\ ODE, UK \\ 3 Theoretische Physik I, Heinrich Heine Universität, 40225, Düsseldorf, Germany \\ ${ }^{4}$ UNIST, Ulsan, 689-798, South Korea \\ ${ }^{5}$ Lawrence Livermore National Laboratory, Livermore, CA, 94550, USA \\ ${ }^{6}$ Department of Physics, Capital Normal University, Beijing, 100048, China \\ ${ }^{7}$ Instituto Superior Técnico, Universidade de Lisboa, Lisbon, Portugal \\ E-mail: g.vieux@strath.ac.uk
}

\begin{abstract}
Ultra high power laser amplifier systems based on plasma-based may provide a pathway to reach petawatt to exawatt powers, vastly exceeding the current limitations imposed by the currently low damage threshold of solid state optical elements. In theory unfocused intensities of $10^{17} \mathrm{~W} \mathrm{~cm}^{-2}$ could be reached. The Raman amplification scheme has been demonstrated as a promising candidate through the observation of $10 \%$ energy transfer efficiency due to amplification of noise, which implies potentially much larger efficiencies. However, controlled amplification of a seed pulse has not hitherto exceeded an efficiency of $7 \%$. Here we discuss several saturation mechanisms that can limit the gain, such as early pump scattering and thermal effects. We show that chirped pulse Raman amplification can mitigate these deleterious effects.
\end{abstract}

Submitted to: Plasma Phys. Control. Fusion 


\section{Introduction}

Plasma has been suggested, by Shvets et al. [1], as a novel gain medium for amplifying short laser pulses. It has potential to overcome current limitations of the chirped pulse amplification technique imposed by the low damage threshold of solid state media. This approach, more generally known as Raman amplification, is based on the paradigm of three-wave interaction between two electromagnetic waves and an electron plasma wave [2], which can lead to superradiant amplification of a short seed pulse counterpropagating with respect to a long pump pulse in plasma. For energy exchange to take place the following resonance conditions must be satisfied: $\omega_{0}=\omega_{1}+\omega_{p}$ and $\boldsymbol{k}_{0}=\boldsymbol{k}_{1}+\boldsymbol{k}_{p}$, where $\omega_{0,1, p}, \boldsymbol{k}_{0,1, p}$ are the frequencies and wave-vectors of the pump, seed and plasma waves, respectively. When pump depletion is negligible the seed pulse amplitude grows exponentially in time, with a growth factor given by $\gamma_{0}=a_{0} \sqrt{\omega_{0} \omega_{p}} / 2$, for a linearly polarized wave, which is known as the linear regime. Here, $a_{0}$ represents the normalized vector potential of the pump beam. In this regime, the duration of the seed pulse increases while its amplitude maximum propagates at $c / 2$ and its front travels at $c$, the speed of light, because of the intrinsically narrow gain bandwidth of the Raman instability. As pump depletion becomes significant the front of the seed pulse absorbs most of the pump energy, while the growth of the back of the seed pulse is strongly suppressed, which defines the non-linear regime. In this case the peak or amplitude maximum propagates with a super-luminal velocity and the amplification process displays characteristics of superradiance [3], which is characterised by: (i) a linear amplitude growth with propagation distance, (ii) a seed duration that decreases inversely proportional to its amplitude, and (iii) a frequency bandwidth that increases with amplitude. As a result, the non-linear instability can tolerate increasingly larger detunings from resonance, which makes the amplification scheme less susceptible to fluctuations in the plasma frequency. A cold plasma theoretical description predicts that the energy transfer efficiency can be as high as $\omega_{1} / \omega_{0} \approx 0.9$, but numerical simulations indicate around 35\% [4]. Of more concern is the large discrepancy with experimental results, where measured energy transfer efficiencies are typically of the order of a percent $[5,6,7,8,9,10,11]$, and for the best results do not exceed $\sim 7 \%$, although pulse compression is observed [12]. Several concurrent phenomena can lead to the saturation of the gain, including early scattering of the pump pulse on noise (fluctuations), Raman forward scattering of the seed pulse, thermal effects leading to Bohm-Gross shifts [13], wavebreaking [14] and particle trapping. In this paper we present what we believe are currently the two main deleterious phenomena and discuss ways of mitigating them. In section 2 early Raman scattering of the pump is discussed, while in section 3 a means of reducing its deleterious effects is introduced in the form of chirped pulse Raman amplification. In section 4 thermal effects are considered before the conclusion. 

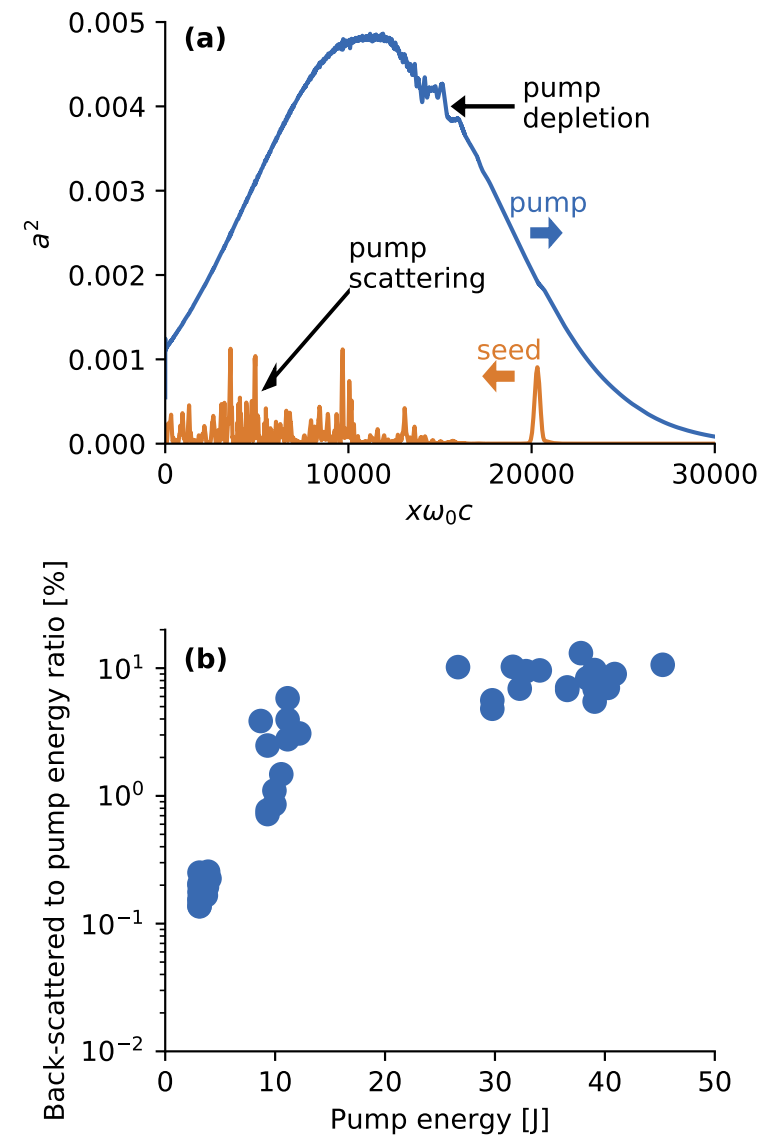

Figure 1. (a) 1D Vlasov simulation showing early pump scattering with signs of pump depletion before the start of seed amplification. Pump parameters are chosen to match an experiment studying chirped pulse Raman amplification (see section 3 ): $a_{0}=0.071$, central wavelength, $\lambda_{0}=800 \mathrm{~nm}$, bandwidth $40 \mathrm{~nm}$, duration $6.5 \mathrm{ps}$ with a positive frequency chirp. (b) Experimental results using a quasi-monochromatic pump. Ratio of back-scattered Raman energy to initial pump energy. Up to $10 \%$ of the pump energy is converted [11].

\section{Early scattering of the pump pulse}

Spontaneous Raman scattering develops from thermal plasma fluctuations and produces light electromagnetic waves that are further amplified prior to the pump interacting with the seed, as illustrated in figure 1(a). When the pump reaches the seed the plasma collective response is already significantly disturbed because of the different saturation mechanisms. Spontaneous scattering depends on the pump intensity and can result in back-scattering of $\sim 10 \%$ of the pump energy, as has been experimentally demonstrated [11], with the main results also shown in figure 1(b), which were obtained by firing a 10-ps long, quasi-monochromatic beam with a central wavelength of $1.053 \mu \mathrm{m}$ into a 3-mm long plasma slab with a density $6-7 \times 10^{18} \mathrm{~cm}^{-3}$. Here, the pump intensities range from $10^{14}$ to $\sim 5 \times 10^{15} \mathrm{Wcm}^{-2}$ and for an pump energy of $50 \mathrm{~J}$, up to $5 \mathrm{~J}$ is back-scattered. Moreover, studies comparing low-intensity seed amplification 
with amplification from noise in a non-counterpropagating geometry, show that as the pump intensity increases to $10^{16} \mathrm{~W} \mathrm{~cm}^{-2}$, the collected energies reach the same order of magnitude [11].

To suppress Raman instability of the pump to noise it has been suggested that a plasma density gradient is combined with a pump frequency chirp [15]. In this case the plasma frequency gradient suppresses Raman forward scattering of the seed, while the pump frequency chirp suppresses pump Raman back-scattering from noise. The two gradients can be chosen to keep the seed in resonance as it propagates through the plasma and the pump.

\section{Chirped pulse Raman amplification}

As a first step we have studied Chirped Pulse Raman Amplification (CPRA) including only the effect of the pump frequency chirp on the interaction [16]. The general principle is schematically presented in figure 2 . In the linear regime, under the resonance condition, the gain is distributed along the plasma, with each seed spectral component being amplified in turn over a limited time $\tau=\pi \gamma_{0} / 2 \alpha$, where $\alpha$ is the linear frequency chirp rate of the pump. Here, $\tau$ represents the time to pass through the resonant bandwidth $\sim \pi\left|\gamma_{0}\right|$ (referred as the amplification band in figure 2). This limits the achievable gain to $G=\exp \left(\frac{\pi \gamma_{0}^{2}}{2 \alpha}\right)$ and limits the amplitude of the plasma wave. It also reduces losses due to wave breaking, as will be explained in the following section. Interestingly, CPRA displays similar characteristics to superradiant amplification, i.e., amplitude growth linear with the propagation distance and self-similar contraction as illustrated in figure 2, where 3 snapshots of the seed, obtained from a numerical simulation, are presented: initial seed, amplified seed after propagation through half and the full duration of the pump pulse, respectively. Evolution of the spectrum is also presented. CPRA can therefore easily be confused with the non-linear regime. It has been demonstrated in early experiments, in the linear regime, using moderate pump and seed intensities, with the beams propagating in a long plasma channel produced in a capillary discharge waveguide [9]. As illustrated in figure 3, a typical energy gain of around $400 \%$ over the full spectral bandwidth is observed with an energy transfer efficiency of $1 \%$. The initial short pulse duration is preserved and no scattering from noise is observed (within the dynamic range of the CCD camera imaging the output of the capillary) because the pump intensities are kept below $10^{14} \mathrm{~W} \mathrm{~cm}^{-2}$ and the plasma density is low $\left(\leq 2 \times 10^{18} \mathrm{~cm}^{-3}\right)$. These results have been obtained using a 45 $\mathrm{nm}$ bandwidth, 300-ps, 350-m J pump beam and a 200-fs, $250-\mu \mathrm{J}$ seed beam interacting in a 4-cm long plasma channel with an electron density of $2 \times 10^{18} \mathrm{~cm}^{-3}$. Both beams, of waist $\approx 60 \mu \mathrm{m}$ at the entrances of the capillary, are circularly polarized, which has the advantage of limiting inverse bremsstrahlung heating.

As a singular measure, CPRA can prevent excessive growth of spontaneous scattering in the linear regime but it remains to be demonstrated that this still allows the seed to evolve in the non-linear regime if a high enough intensity is achieved. Recently, an 


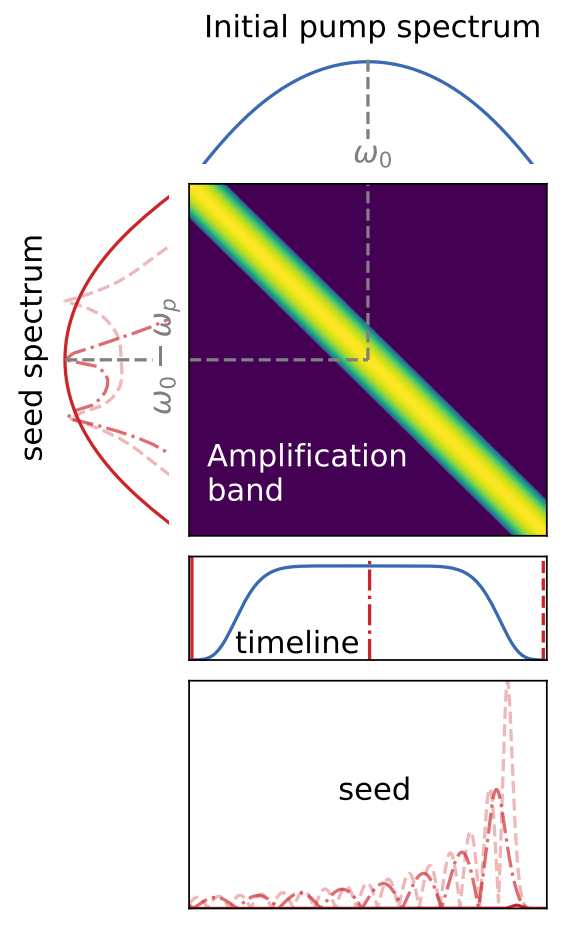

Figure 2. Principle of Chirped Pulse Raman Amplification (CPRA). The gain is distributed along the plasma with each seed spectral component being amplified at a different time and position, giving rise to an amplification band with width $\sim \pi\left|\gamma_{0}\right|$ in the frequency domain and duration $\pi \gamma / 2 \alpha$ in the time domain. CPRA exhibits the same characteristics as the non-linear regime, as observed in the simulation snapshots.

experimental campaign was conducted at the Central Laser Facility at RAL using the Astra-Gemini laser system, to investigate CPRA in the non-linear regime. In these experiments a frequency chirped pump $\left(\alpha \approx 2 \times 10^{25} \mathrm{rad} \mathrm{s}^{-2}\right)$ with an intensity and duration similar to that used in Vieux et al., [11] is used. The seed pulse has an intensity of $10^{15} \mathrm{~W} \mathrm{~cm}^{-2}$, with energy $\sim 13 \mathrm{~mJ}$ and initial seed duration $150 \mathrm{fs}$. It is first observed that scattering from noise cannot be avoided at high intensities, as illustrated in figure 4 where the normalised measurements of scattering from noise for both the quasi-monochromatic and chirp cases are presented. Similar Raman signal levels are obtained for intensities above $10^{15} \mathrm{~W} \mathrm{~cm}^{-2}$ and for equivalent densities. However, it is also observed that the seed grows faster than the noise. Table 1 summarises the signal-to-noise $(\mathrm{S} / \mathrm{N})$ ratios measured for the highest pump intensity $\left(\sim 10^{16} \mathrm{~W} \mathrm{~cm}^{-2}\right)$ during our last campaign together with the previously measured ratios using a quasimonochromatic pump pulse. The chosen pressures satisfy the resonance conditions, apart from the entry at 28 bar, which is the highest pressure used and is given solely as an indication. Figure 5 presents the energy measurements from which the $\mathrm{S} / \mathrm{N}$ ratios for the chirped case are deduced. While it is not possible to conduct a strict one- 


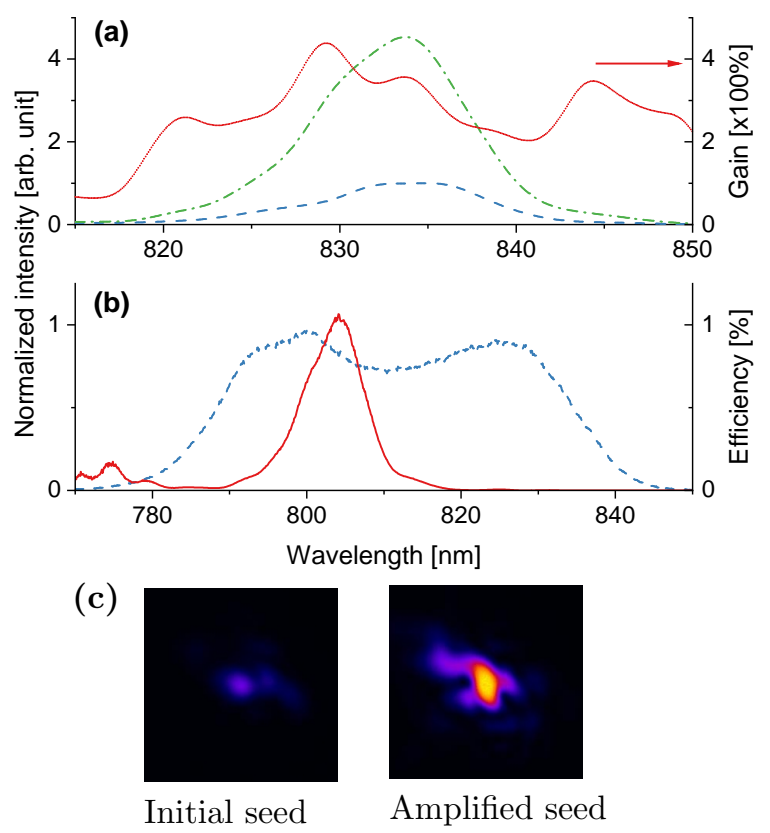

Figure 3. Typical result demonstrating CPRA in the linear regime and long plasma medium. Main pump parameters: bandwidth, $45 \mathrm{~nm}$, duration, $300 \mathrm{ps}$, energy, $350 \mathrm{~mJ}$, chirp rate, $4.5 \times 10^{23} \mathrm{rad} \mathrm{s}^{-2}$. (a) initial seed spectrum (blue dash), amplified spectrum (green dot-dash), measured gain (red dot); (b) initial pump spectrum (blue dash), efficiency (red, solid line); (c) seed beam at the output of the capillary, before and after amplification.

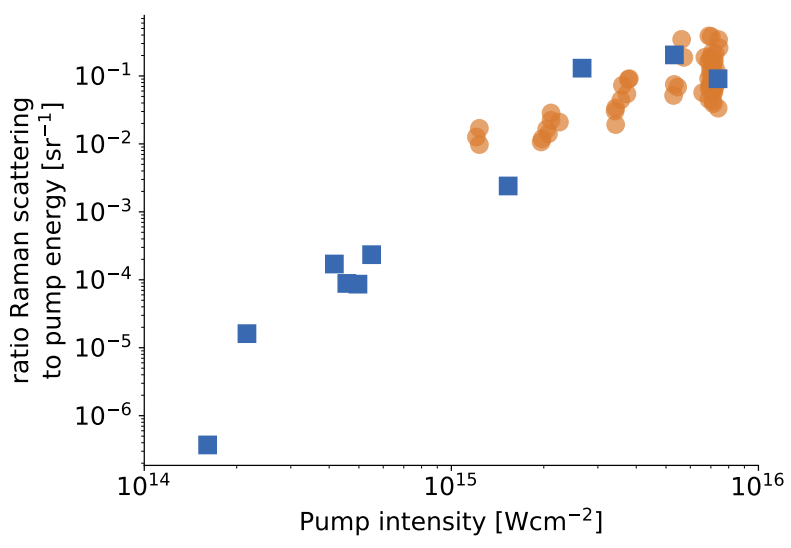

Figure 4. Ratio of noise amplified Raman signal energy to pump energy in the direction of seed propagation. The values are normalised to take into account the light collection solid angle. (Square, blue) quasi-monochromatic pump, (circle, orange) frequency chirped pump. 


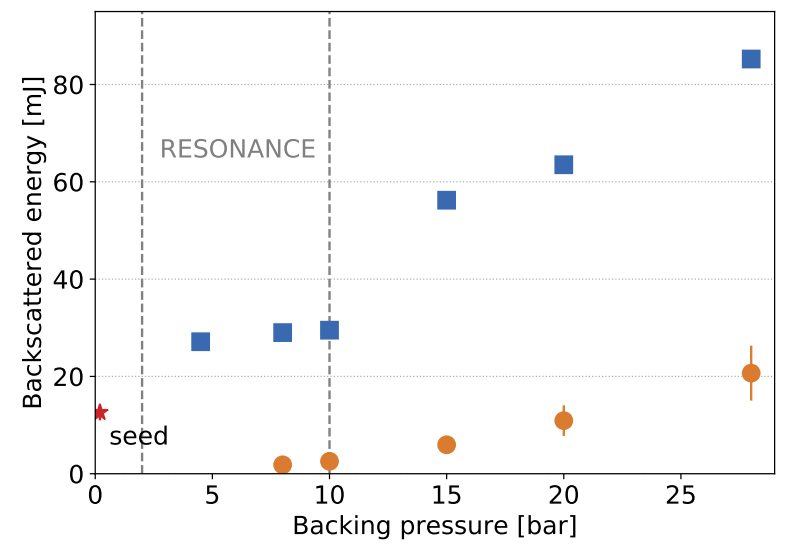

Figure 5. Back-scattered energy measurements using intense pump and seed pulses. (Circle, orange) Raman scattering from noise; (square, blue) Raman signal with seed.

Table 1. Raman signal-to-noise $(\mathrm{S} / \mathrm{N})$ ratios for a chirped and a quasi-monochromatic pump pulse and different densities. S/N ratios are calculated as follows: (S-I)/N and in brackets $(\mathrm{S}-\mathrm{I}-\mathrm{N}) / \mathrm{N}$, where $\mathrm{S}$ is the measured signal energy when the seed pulse is injected, $\mathrm{N}$ is the amplified noise signal and $\mathrm{I}$ is the initial seed energy.

\begin{tabular}{|c|c|c|c|c|c|}
\hline \multirow[b]{2}{*}{ pressure [bar] } & \multicolumn{4}{|c|}{ frequency chirp } & \multirow{2}{*}{$\frac{\text { monochromatic }}{12}$} \\
\hline & 4.5 & 8 & 10 & 28 & \\
\hline $\mathrm{S} / \mathrm{N}$ ratio & $23.3(22.3)$ & $9(7.9)$ & $6.6(5.6)$ & $3.9(2.9)$ & $2.4(1.4)$ \\
\hline
\end{tabular}

to-one comparison, because of (i) the difference in seed intensities, (ii) pump beam energies and quality, (iii) slight difference in beam crossing angle, it is still observed that high $\mathrm{S} / \mathrm{R}$ ratios are obtained in the CPRA regime, which tends to show that the frequency chirp does not significantly alter the seed growth in the Raman nonlinear regime. Interestingly, the highest seed energy is obtained for a highly detuned interaction with the gain increasing with plasma density, as illustrated in figure 5. For densities where the resonance condition is satisfied $\left(1-5 \times 10^{18} \mathrm{~cm}^{-3}\right)$, amplification of a factor of 2 is observed, while it is almost 6 for a density of $1-2 \times 10^{19} \mathrm{~cm}^{-3}$. Initial particle-in-cell simulations suggest that pump energy is scattered by the plasma electrons and not by the ions, as it would be the case from Brillouin scattering. This supports our current hypothesis that the amplifier operates in the nonlinear Raman regime, where the electron motion is dominated by the ponderomotive potential of the beatwave [1].

\section{Thermal effects}

As mentioned above, the plasma finite temperature also limits the seed amplitude growth by (i) reducing the Raman growth rate by a factor $\left(1+12 \beta_{t h}^{2} \omega_{0}^{2} / \omega_{p}^{2}\right)^{-1 / 4}$, where $\beta_{t h}$ is the thermal velocity of the electrons, $v_{t h}$, normalized to the speed of light [10]; (ii) 
introducing a plasma frequency shift and reducing the plasma wave amplitude through the Bohm-Gross shift and particle trapping [13, 17]; (iii) lowering the threshold to reach wave breaking $[14,18]$, which suppresses the collective plasma response [10]. It should be noted that while small amplitude plasma waves are damped only by electrons with a velocity close to the wave phase velocity, $v_{p h}$, this is no longer the case for a large amplitude wave. In this situation, the electron oscillation velocities in the field is sufficiently large to bring particles from an initially cold plasma into resonance with the plasma wave. For wave breaking to occur, i.e. for a plasma wave amplitude, $E_{w b}$, such that $\left(e E_{w b} / m \omega\right) \approx \omega / k=v_{p h}$, a large number of formerly non-resonant electrons become trapped, resulting in strong nonlinear damping of the plasma wave $[2,19]$. The value of $E_{w b}$ decreases for a warm plasma [14], as shown in figure 6(a). Energetic electrons are more easily brought into resonance with the wave and the pressure force associated with the density fluctuation of the wave gives an additional acceleration. Figure 6(a) is obtained from Coffey's waterbag model [14]. The decrease in the maximum attainable field amplitude as function of the parameter $v_{t h} / v_{p h}$ is rapid. For $v_{t h} \approx v_{p h} / 2$ the plasma wave is fully damped. This suggests that moderately high electron density is required, since the phase velocity increases with the density as illustrated in figure 6(b). Those observations reinforce the proposition to use a moderately intense pump with a frequency chirp, which limits the growth of a plasma wave before pump-seed interaction and therefore avoids early wave breaking of the Langmuir wave.

Thermal effects on the Raman amplification process in the linear regime have been indirectly observed in studies of the effect of the seed frequency chirp on amplification [10]. Amplification factors for seed pulses of energy $750 \mu \mathrm{J}$ and durations from $120 \mathrm{fs}$ to $400 \mathrm{fs}$ have been measured under different pump energies. A positive chirp consistently resulted in a higher amplification factor, usually by a factor $\sim 2$. One explanation is that, due to the large seed chirp rate, the beat-wave excites a plasma wave with a rapidly varying phase velocity along the seed temporal envelope, with $v_{p h}$ increasing (decreasing) for negative (positive) chirp. For our parameters, Coffey's theory [14] indicates that the plasma wave is heavily damped at the front of the negatively chirped seed because $v_{p h}$ is lower than the thermal velocity. The plasma wave amplitude has therefore less time to grow, leading to saturation. In the case of the positively chirped seed, the plasma wave is excited initially from the front of the pulse, since $v_{p h}>v_{t h}$, which extends the period of growth, even when damping of the plasma wave occurs at later times because of the reduction in $v_{p h}$ [10].

\section{Conclusion}

Raman amplification in plasma is a promising approach to producing ultra-high power laser pulses. However, a number of deleterious phenomena still need to be controlled to make it an efficient scheme. We have discussed two limiting factors, early scattering of the pump and thermal effects, which can suppress the necessary collective plasma response. Raman instability of the pump to noise can be mitigated by using a pump 

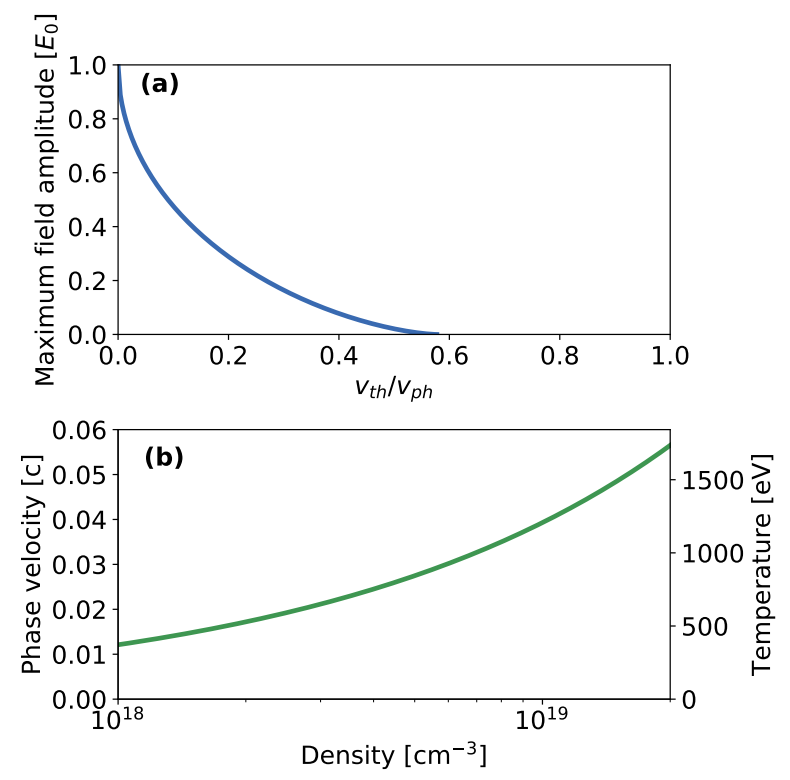

Figure 6. (a) Wave breaking threshold of the plasma wave amplitude. Value given as the ratio relative to the cold plasma value. (b), Phase velocity of the longitudinal plasma wave as function of the plasma density. The corresponding temperatures are also presented.

beam with moderate intensity combined with a frequency chirp. Growth of the noise is suppressed because of the rapid frequency detuning in the linear regime, while a seed with a sufficently high amplitude can still grow in the non-linear regime. When the pump intensity becomes large the beneficial impact of the frequency chirp diminishes and the noise signals are similar to those recorded for the unchirped case. CPRA experimental results have shown that it is feasible to obtain amplification of a seed pulse through a non-resonant interaction when the seed is intense. Interestingly, Raman signal energies measured during our experimental campaign using a quasi-monochromatic pump with a resonant interactions [11] are comparable with our latest results on CPRA. However the latter have been obtained at a fraction of the pump energy, (3.5 J, compared with $70 \mathrm{~J}$ ), which leads to lower scattering from noise. Thermal effects also contribute to gain saturation through particle trapping and wave breaking. Here also CPRA can act positively by limiting the amplitude of the plasma wave and therefore particle trapping. Moreover, the phase velocity of the plasma wave must be chosen to be larger than the thermal velocity of the electrons, which requires moderately high plasma densities. Numerically, a set of parameters have been identified that leads to robust amplification [10], but this is still experimental work in progress.

\section{Acknowledgments}

We acknowledge support of the U.K. EPSRC (grant no. EP/N028694/1), the EC's Laserlab-Europe, H2020 EC-GA 654148 and ELI - Extreme Light Infrastructure phase 
2 (CZ.02.1.01/0.0/0.0/15_008/0000162) from European Regional Development Fund. MSH is supported by the National Research Foundation of Korea (grant numbers NRF2017M1A7A1A03072766). Simulation results have been obtained using the EPSRC funded ARCHIE-WeSt High Performance Computer (www.archie-west.ac.uk).

6

Data associated with research published in this paper is accessible at http://dx.doi.org/10.15129/2fd625bf-2d54-4514-a664-04876145f6a8 and http://dx.doi.org/10.15129/a55d0576-fdff-4a0f-b0da-70e025523c7a.

\section{References}

[1] Shvets G, Fisch N J, Pukhov A and ter Vehn J M 1998 Phys. Rev. Lett. 814879

[2] Kruer W 1988 The Physics of Laser Plasma Interaction (Addison-Wesley, Reading, MA)

[3] Malkin V M, Shvets G and Fisch N J 1999 Phys. Rev. Lett. 824448

[4] Trines R M G M, Fiúza F, Bingham R, Fonseca R A, Silva L O, Cairns R A and Norreys P A 2011 Nat. Phys. 787

[5] Balakin A A, Kartashov D V, Kiselev A M, Skobelev S A, Stepanov A N and Fră̈man G M 2004 JETP Lett. 8012

[6] Ping Y, Geltner I and Suckewer S 2003 Phys. Rev. E 6716401

[7] Ping Y, Cheng W, Suckewer S, Clark D S and Fisch N J 2004 Phys. Rev. Lett. 92175007

[8] Dreher M, Takahashi E, Meyer-ter Vehn J and Witte K J 2004 Phys. Rev. Lett. 9395001

[9] Vieux G, Lyachev A, Yang X, Ersfeld B, Farmer J P, Brunetti E, Issac R C, Raj G, Welsh G H, Wiggins S M and Jaroszynski D A 2011 New Journal of Physics 13063042

[10] Yang X, Vieux G, Brunetti E, Ersfeld B, Farmer J P, Hur M S, Issac R C, Raj G, Wiggins S M, Welsh G H, Yoffe S R and Jaroszynski D A 2015 Scientific Reports 513333

[11] Vieux G, Cipiccia S, Grant D W, Lemos N, Grant P, Ciocarlan C, Ersfeld B, Hur M S, Lepipas P, Manahan G G, Raj G, Reboredo Gil D, Subiel A, Welsh G H, Wiggins S M, Yoffe S R, Farmer J P, Aniculaesei C, Brunetti E, Yang X, Heathcote R, Nersisyan G, Lewis C L S, Pukhov A, Dias J M and Jaroszynski D A 2017 Scientific Reports 72399

[12] Ren J, Cheng W, Li S and Suckewer S 2007 Nat. Phys. 3732

[13] Bohm D and Gross E P 1949 Phys. Rev. 751851

[14] Coffey T P 1971 Phys. Fluids 141402

[15] Malkin V M, Shvets G and Fisch N J 2000 Physical Review Letters 841208

[16] Ersfeld B and Jaroszynski D A 2005 Phys. Rev. Lett. 95165002

[17] Ersfeld B, Farmer J, Raj G and Jaroszynski D A 2010 Phys. Plasmas 1783301

[18] Farmer J P, Ersfeld B and Jaroszynski D A 2010 Phys. Plasmas 17113301

[19] Dawson J M 1959 Physical Review 113383 